\title{
A hybrid technology for miniaturised inductive device applications
}

\author{
E. Belloy *, S.E. Gilbert, O. Dezuari, M. Sancho, M.A.M. Gijs \\ Room BM3.130, EPFL-DMT-IMS, Institute of Microsystems, Swiss Federal Institute of Technology Lausanne, CH-1015 Lausanne EPFL, Switzerland
}

Accepted 1 November 1999

\begin{abstract}
We have fabricated two-dimensional inductive devices by integrating micropatterned magnetic foils of high permeability $\left(\mu_{\mathrm{r}}=100000\right)$ with winding patterns realised in printed circuit boards (PCBs) and flex-foil technology. We realise relatively high inductances of 1-10 $\mu \mathrm{H}$ for transformer type devices and a sensitivity of $60 \mathrm{~V} / \mathrm{T}$ at $30 \mathrm{kHz}$ for fluxgate magnetic induction sensing devices. The same type of device is also used as a current sensor; here we typically find sensitivities of $10 \mathrm{mV} / \mathrm{A}$ for excitation currents of a few $100 \mathrm{~mA}$. We think our technology opens the way to a new class of functional and economic sensing devices. C 2000 Elsevier Science S.A. All rights reserved.
\end{abstract}

Keywords: Planar transformer; Fluxgate sensor; Thin inductive devices; Current sensor; PCB technology; Flex-foil technology

\section{Introduction}

Inductive and transformer-like devices are at the heart of numerous sensing and actuating applications. With the increasing trend of miniaturisation of electronic devices, often the inductive components are the determining factor limiting further size reduction. Both for the realisation of high inductance transformers $[1,2]$ and for high resolution magnetic or current sensing devices based on inductive measurement principles like fluxgate sensors [3,4], the availability of a high permeability and low hysteresis ferromagnetic core material is essential. We have developed a method for the fabrication of planar microtransformers [5] and fluxgate sensing devices [6] using a hybrid printed circuit board (PCB)/flexible foil technology, where we integrate $\mathrm{Cu}$ winding patterns with magnetic foil core materials. The magnetic core materials used (Vitrovac ${ }^{\circledR}$ 6025 or Metglas ${ }^{\circledR} 2714 \mathrm{~A}$ ) have an extremely high relative magnetic permeability $\left(\mu_{\mathrm{r}}=100000\right)$ and a fairly large thickness $(25 \mu \mathrm{m})[7,8]$. This permeability is one or two orders of magnitude larger than the corresponding properties of films made using classical vacuum deposition methods. Consequently, for a given inductor size, the inductance value can be much higher than obtainable with these methods. Also for magnetic induction and current sensing

\footnotetext{
* Corresponding author. Tel.: +41-21-693-6759; fax: +41-21-6935950.

E-mail address: eric.belloy@epfl.ch (E. Belloy).
}

devices, the sensor's response is proportional to the relative permeability of the core material, hence, also here a high $\mu_{\mathrm{r}}$ is beneficial. Moreover, in our technology, the core material can be selected from a variety of commercially available amorphous magnetic foils, amenable to lithographic patterning.

In this paper, we discuss the fabrication method of these devices and briefly review their application as inductors or as sensitive magnetic field fluxgate sensors. We elaborate more on a third application we recently introduced, which is current sensing. To realise a current sensor, we provide the magnetic fluxgate sensors with a hole in the centre of the PCB structure for the accommodation of a current carrying wire. It is the magnetic field generated by the current through this wire, which generates a second harmonic voltage over the excitation coil, in which we apply an excitation current of a few $100 \mathrm{~mA}$ at frequencies of several $10 \mathrm{kHz}$. By providing 'gaps' in the magnetic core, we can tune the effective permeability and hence the sensitivity of the current sensor. A less sensitive current sensor evidently is operational up to much higher DC current levels. We have measured the immunity of our sensor to its orientation with respect to the earth's magnetic field. We typically find angular dependent variations of a few percent on the output signal for our present current sensor design. Finally, we briefly discuss an etching-electroplating method for the realisation of $\mathrm{Cu}$ coils with high $\mathrm{Cu}$ filling factor, leading to an enhanced electrical quality factor in the frequency regime below $100 \mathrm{kHz}$. 


\section{Fabrication procedure}

The fabrication procedure of our inductive devices has already been described in detail in Refs.[5,6]. The structures are fabricated based on conventional PCB and flexfoil processes. A schematic diagram of the layer built-up is shown in Fig. 1a. Two copper-clad epoxy boards or polyimide foils are used as the outer layers. A liquid epoxy or acrylic adhesive was used to glue Vitrovac ${ }^{\circledR} 6025$ or Metglas ${ }^{\circledR}$ 2714A foil on the interior non-copper-bearing support. These foils were patterned photolithographically to form the transformer cores. For the transformers and magnetic field fluxgate sensors, we have used Vitrovac ${ }^{\circledR}$ 6025 foils, while for the fluxgate-type current sensing devices, we have used the Metglas ${ }^{\circledR}$ 2714A. Coil patterns were printed onto the copper laminates using standard photolithographic methods. Alternate half-windings were structured into each outer laminate layer, and corresponding top and bottom coil windings were connected by copper-filled vias. PCB-based structures were finally as- sembled and bonded by hot pressing with Prepreg ${ }^{\circledR}$ epoxy sheets, whereas for the flex-foil structure, we used acrylic adhesive layers. Photographs of the realised PCB transformers and of a PCB and flex-foil fluxgates are presented in Fig. $1 b$ and c,d, respectively.

\section{Inductive devices}

\subsection{Transformers}

As an example, we show in Fig. 2 the frequency dependence of the primary self-inductance, $L$, and the coil resistance, $R$, measured on a 10 turn primary rectangular transformer with an open secondary winding (a device of the type shown in Fig.1b). Vitrovac ${ }^{\circledR} 6025$ material was used as the core material. The self-inductance $L$ attains its highest values at low frequencies, and in this case a maximum of about $3.5 \mu \mathrm{H}$ is measured at $1 \mathrm{kHz}$. The inductance characteristic mainly follows the intrinsic per-
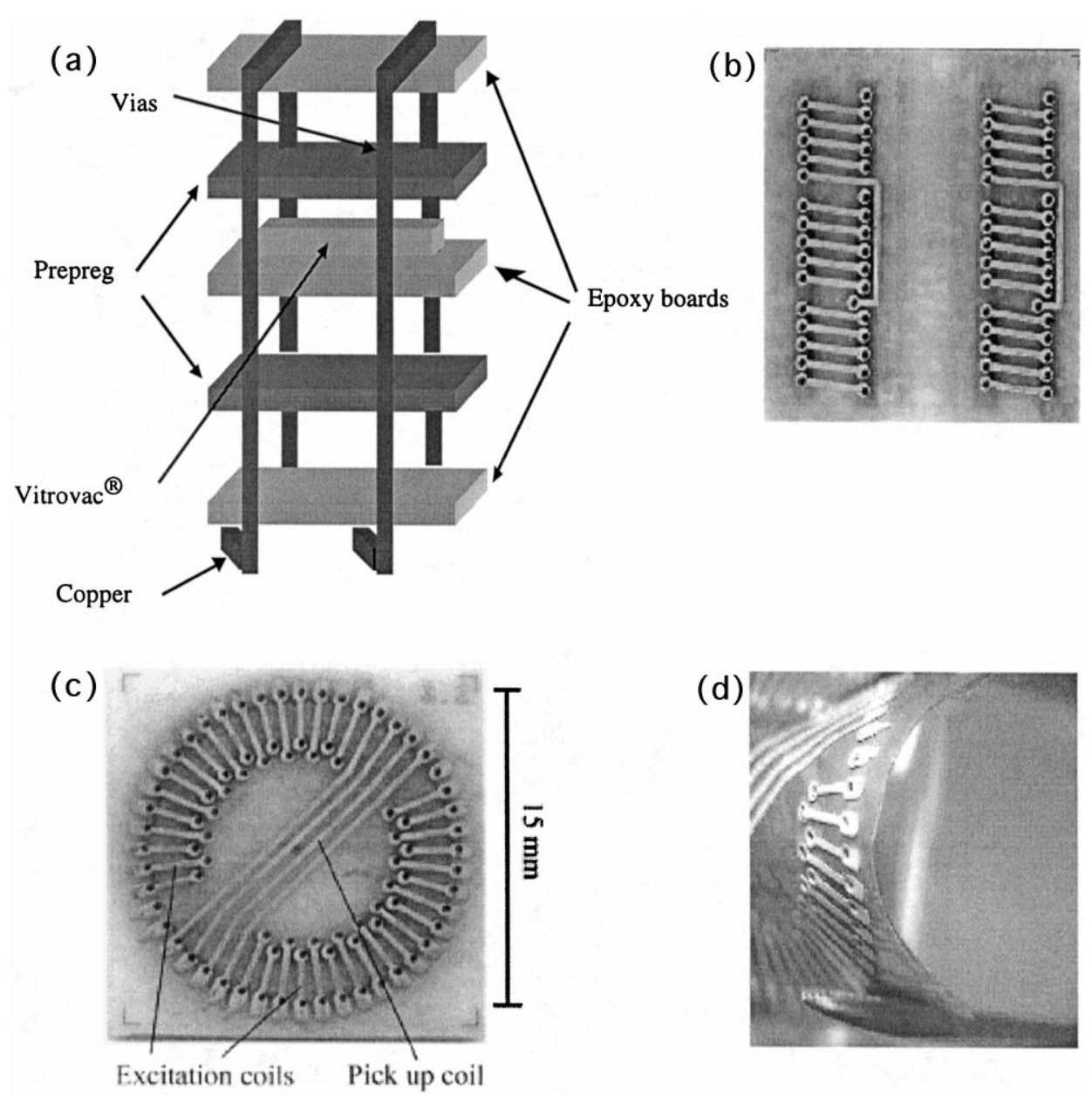

Fig. 1. (a) Schematic diagram of the inductive devices fabrication process; (b) photograph of rectangular PCB transformers, characterised by 10 primary and 5 secondary windings; (c) photograph of a toroidal PCB fluxgate with two excitation and one pick up coil; (d) photograph of a toroidal flex-foil fluxgate. 


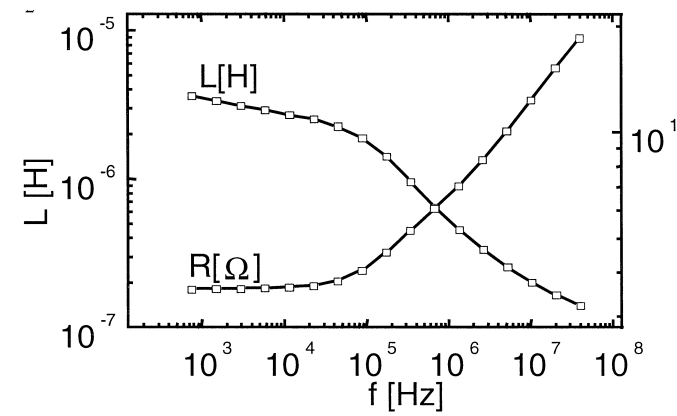

Fig. 2. Inductance and resistance as a function of frequency for a rectangular transformer.

meability dependence of the Vitrovac ${ }^{\circledR} 6025$ material. The rise in coil resistance starting at about $50 \mathrm{kHz}$ is due to eddy current and magnetic core losses. A more detailed discussion of the realisation and characterisation of our transformer devices can be found in Ref. [5].

\subsection{Fluxgate magnetic field sensors}

The principle of a fluxgate is the external magnetic field-dependent periodic saturation of a ferromagnetic material. Soft magnetic alloys like Vitrovac ${ }^{\circledR}$ or Metglas ${ }^{\circledR}$, having a small hysteresis and a large saturation magnetisation are well known for their use as core material. The combined action of the external field (to be measured) and excitation coils, driving the ferromagnetic core in saturation, leads to the generation of higher order harmonics of the fundamental excitation frequency in a detection coil surrounding the magnetic core. The second harmonic voltage $V_{2 \mathrm{f}}$ can be filtered out of the detection signal using a lock-in technique and is proportional to the external field for a certain field range (typically 0.01-100 $\mu \mathrm{T}$ ). Two different types of fluxgate sensors have been designed and fabricated: the first type has the inner magnetic foil patterned into a toroidal shape and the second type has a magnetic core of rectangular shape. A photograph of a toroidal structure was displayed in Fig. 1c. One should note that the two excitation coils are positioned symmetrically at both sides of the detection coil. Each excitation coil has 18 windings while only four were used for the detection coil.

Fig. 3 represents the second harmonic voltage amplitude $V_{2 \mathrm{f}}$ of the detection coil of a rectangular fluxgate sensor without air gap in the Vitrovac ${ }^{\circledR}$ magnetic material, as a function of the external magnetic induction $B_{\text {ext }}$ for various excitation currents $I_{\mathrm{e}}$ at $10 \mathrm{kHz}$.. We have chosen the direction of $B_{\text {ext }}$ in the line of the horizontal component of the local terrestrial magnetic field (in Switzerland $B_{\text {terrestrial }} \approx 20 \mu \mathrm{T}$ ). One notes that for all excitation currents, $V_{2 \mathrm{f}}$ approaches zero at this field; this situation corresponds to an effective zero magnetic field. One notes that for all $I_{\mathrm{e}}$, there is a linear dependence on $B_{\text {ext }}$ in the low field range. This range becomes more extended for the higher excitation currents. The symmetric response around the true zero field with respect to the sign of the external magnetic field is immediately clear. Moreover, the curves shown in Fig. 3 represent data taken by increasing the external field from -250 to $250 \mu \mathrm{T}$ and vice versa. Within the limit of our detection, we found no evidence of any hysteretic behaviour. Concluding, we obtain rather high sensitivities with our devices (up to $60 \mathrm{~V} / \mathrm{T}$ at 30 $\mathrm{kHz}$ ). A more detailed study of these magnetic fluxgate sensors was presented in Ref. [6].

\subsection{Fluxgate-based current sensors}

Our technology equally well can be used for the realisation of current sensors; in this case, we measure the magnetic field generated by a current through a wire, which is inserted inside a hole at the central point of the sensor. Fig. 4a is a photograph of the layout of one type of device. It consists of two excitation coils consisting of 18 windings each centred around a PCB embedded ring patterned out of the Metglas ${ }^{\circledR} 2714$ material. This patterned core can be simple, as shown in Fig. 4b, or can contain 'gaps', as shown in Fig. 4c, to diminish sensitivity and to extend the current measurement range of the device. For the measurement of a DC current, we apply an excitation current in the two coils in series and measure the second harmonic voltage amplitude over the same coil. Clearly, a higher DC current introduces a larger asymmetry in the saturation characteristics of the magnetic material, hence, a large second harmonic component. The central coil, consisting of four windings, is the analogue of the detection coil for the fluxgate magnetic field measurements, and is not used for the current sensing experiments.

Two curves of an experimental current measurement are shown in Fig. 5a,b for a sensor, having a gap-free magnetic core material, as shown in Fig. 4b. We present here

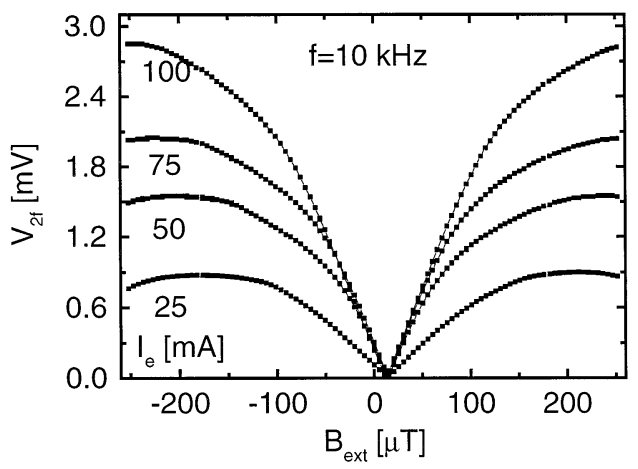

Fig. 3. Second harmonic voltage $V_{2 f}$ of the detection coil of a rectangular fluxgate sensor as a function of $B_{\text {ext }}$ for various excitation currents $I_{\mathrm{e}}$ at $10 \mathrm{kHz}$. 
(a)

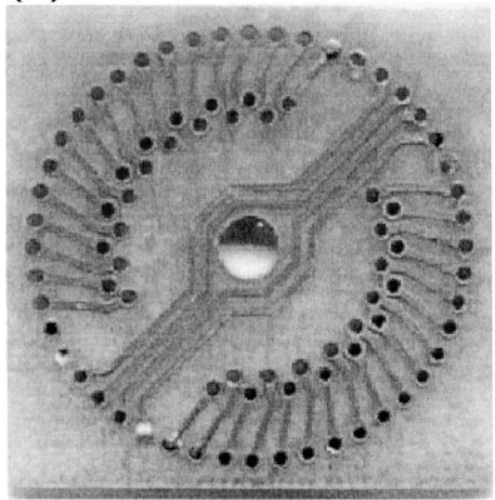

(b)

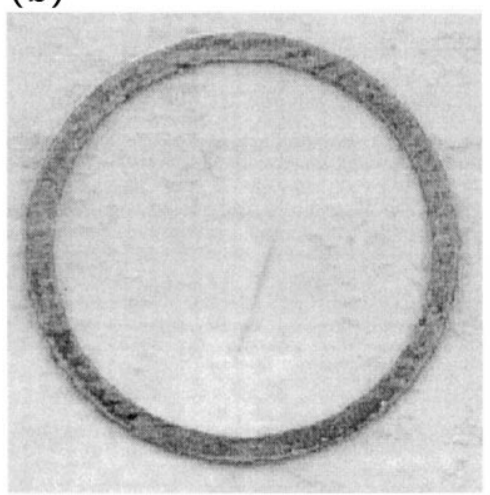

(c)

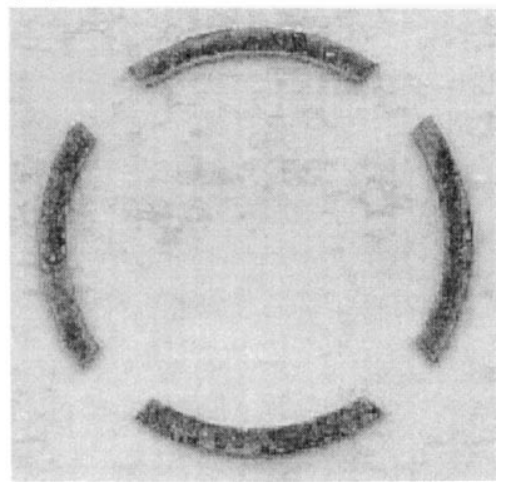

Fig. 4. Photographs of (a) the experimental realisation of a current sensor based on the hybrid PCB technology, (b) a micropatterned Metglas ${ }^{\circledR}$ core without gaps in the magnetic material, to be embedded inside the PCB, (c) a micropatterned Metglas ${ }^{\circledR}$ core with four gaps of $3 \mathrm{~mm}$ in the magnetic material.

the second harmonic voltage amplitude $V_{2 \mathrm{f}}$ versus DC current $I_{\mathrm{DC}}$ through a wire placed within the hole of the sensor. Fig. 5a represents curves measured at three different excitation frequencies with rms amplitude of $175 \mathrm{~mA}$ of the excitation current. We observe a similar characteristic as with the magnetic field fluxgate measurements, i.e., a linear-like zone at small $I_{\mathrm{DC}}$, hence small field, followed by a maximum and subsequently a decrease of the sensor's response. Also with increased frequency, the sensor output is enhanced. The measured sensitivity of the sensor is about $10 \mathrm{mV} / \mathrm{A}$. When using an rms excitation current of $350 \mathrm{~mA}$ (Fig. 5b), we obtain a somewhat different behaviour, as we only observe the initial part of the $V_{2 \mathrm{f}}-I_{\mathrm{DC}}$ response curve of Fig. 5a. The explanation is simple; the sensor will have a maximum response when the absolute value of the magnetic field induced by the AC current is of order of the magnetic field of the DC current to be measured. The use of higher excitation currents automatically results in the extension of the useful regime of the sensor to higher currents.

Fig. 6 shows the characteristics of a current sensor with the embedded patterned core material of Fig. 4c, also at three different excitation frequencies and for an excitation current of $175 \mathrm{~mA}$. We immediately observe the much smaller output of this sensor. Indeed, by providing gaps within the core material, we have strongly decreased the effective permeability of the coil interior and thereby strongly reduce the magnitude of the magnetic fields generated by the various currents. We attribute the asymmetry of the curves to the non-perfect symmetry of the windings and magnetic core material shape, and the non-zero value of $V_{2 \mathrm{f}}$ at $I_{\mathrm{DC}}=0$ to the contribution of the earth's magnetic field of the sensor. The latter is expected to have some influence as our excitation coil consists in fact of

(a)

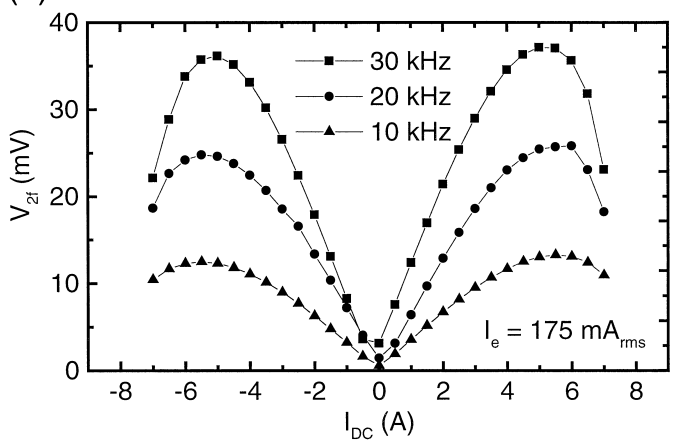

(b)

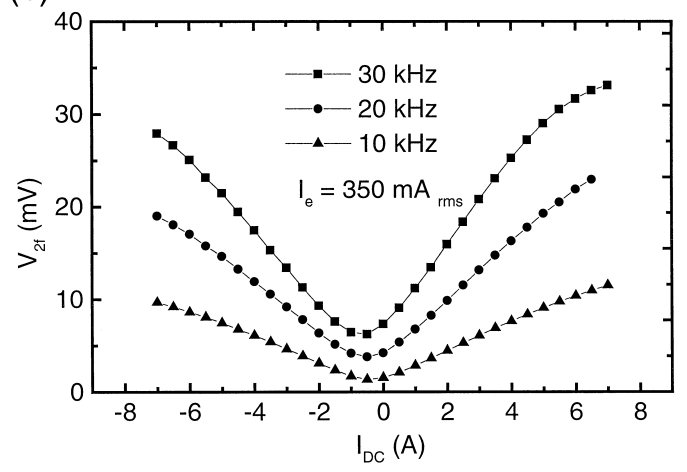

Fig. 5. Second harmonic voltage amplitude $V_{2 \mathrm{f}}$ as a function of applied DC current for the current sensor corresponding with the patterned magnetic core of Fig. $4 \mathrm{~b}$ at three different excitation frequencies, (a) using an excitation current $I_{\mathrm{e}}=175 \mathrm{~mA}_{\mathrm{rms}}$, (b) using an excitation current $I_{\mathrm{e}}=350 \mathrm{~mA}_{\mathrm{rms}}$. 


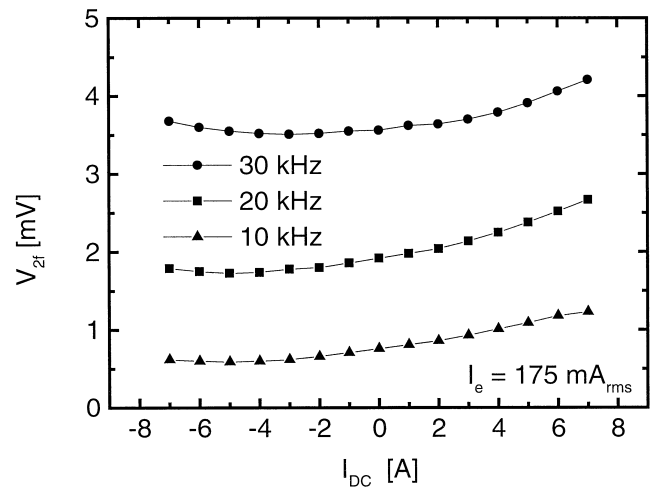

Fig. 6. Second harmonic voltage amplitude $V_{2 \mathrm{f}}$ as a function of applied DC current for the current sensor corresponding with the 'gapped' patterned magnetic core of Fig. 4c at three different excitation frequencies and using an excitation current $I_{\mathrm{e}}=175 \mathrm{~mA}_{\mathrm{rms}}$.

two parts, and due to this symmetry, is susceptible to the direction of the earth field.

The second harmonic voltage amplitude $V_{2 \mathrm{f}}$ as a function of the positioning of the gapped sensor with respect to the earth's magnetic field, is shown in Fig. 7. The excitation is at $30 \mathrm{kHz}$ with rms current value of $350 \mathrm{~mA}$. The position of the sensor at $0^{\circ}$ corresponds with the symmetry axis of the sensor aligned with the direction of the earth's magnetic field. The understanding of this field dependence is rather complicated and involves both asymmetry induced by the electrical winding patterns and asymmetry induced by the micropatterning of the four gaps in the magnetic material. A more detailed study of these influences on the orientational dependence of the current sensor's output will be the subject of future research.

\subsection{Increasing the quality $(Q)$ factor}

A new solution to achieve planar low resistance inductive devices is to apply a Cu-electroplating step after

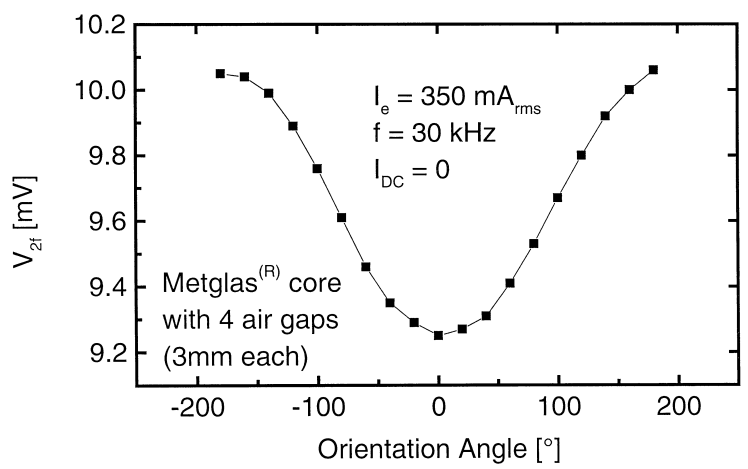

Fig. 7. Earth magnetic field immunity of the current sensor: second harmonic voltage amplitude $V_{2 \mathrm{f}}$ as a function of orientation angle using an excitation current $I_{\mathrm{e}}=350 \mathrm{~mA}_{\mathrm{rms}}$ and for zero DC current $\left(I_{\mathrm{DC}}=0\right.$ A) for the current sensor corresponding with the patterned magnetic core of Fig. 4b.

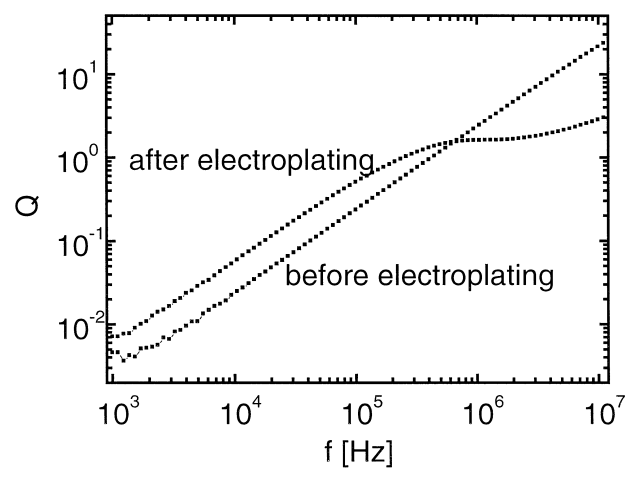

Fig. 8. Comparison of the quality factor of a flat $10 \mu \mathrm{H}$ coil, before and after the electroplating step.

patterning of the windings to enhance the $\mathrm{Cu}$ volume of the windings. Recent experiments on $\mathrm{Cu}$ coils have shown that by using a small electroplating current, it is possible to get a uniform and homogenous $\mathrm{Cu}$ deposition along the coil, increasing its section and minimising the interspacing between the windings. As a result, the quality factor $Q$ can be easily increased by a factor two and the magnetic leakage flux can be decreased substantially. The method works well at lower frequencies; at higher frequencies capacitive coupling between the windings becomes increasingly important. Fig. 8 shows the quality factor $Q=$ $2 \pi f L / R$ of a centimeter-size coil with an inductance of 10 $\mu \mathrm{H}$. We clearly observe a doubling of the quality factor at the lower frequencies. This phenomenon can be exploited for miniaturised low-frequency applications. A more complete study of the use of this etching-electroplating method for the enhancement of the quality factor of inductive devices has been reported elsewhere [9].

\section{Conclusions}

In this paper, we have presented a new technology based on a low cost PCB or flexible foil process. The use of an amorphous metal foil as a magnetic core permits the facile development of high performance inductive devices: inductances of $1-10 \mu \mathrm{H}$ at $1 \mathrm{kHz}$ for the transformers and a sensitivity of up to $60 \mathrm{~V} / \mathrm{T}$ at $30 \mathrm{kHz}$ for the fluxgate. For the current sensor applications, we report sensitivities of typically $10 \mathrm{mV} / \mathrm{A}$. We can tune the sensor sensitivity by changing the shape of the micropatterned magnetic core, changing the frequency or simply changing the number of windings. In comparison with other technologies, our process avoids time consuming and expensive thin film deposition techniques, while retaining compatibility with standard electronic packaging schemes. Therefore, we think that our technology opens the way to a new class of functional and economic sensing devices. 


\section{References}

[1] K. Yamasawa, K. Maruyama, I. Hirohama, P.P. Biringer, High frequency operation of a planar-type microtransformer and its application to multilayered switching regulators, IEEE Trans. Magn. 26 (1990) 1204-1209.

[2] H. Tsujimoto, T. Koiso, Characteristic in the 0.01-2.6 GHz range of film transformer with coils on both sides of flexible polyamide film, IEEE Trans. Magn. 32 (1996) 4980-4982.

[3] F. Primdahl, The fluxgate magnetometer, J. Phys. E: Sci. Instrum. 12 (1979) 241-253.

[4] P. Ripka, Review of magnetic fluxgate sensor, Sens. Actuators, A 33 (1992) 129-134.

[5] O. Dezuari, S.E. Gilbert, E. Belloy, M.A.M. Gijs, A new hybrid technology for planar microtransformer fabrication, Sens. Actuators, A 71 (1998) 198-207.

[6] O. Dezuari, E. Belloy, S.E. Gilbert, M.A.M. Gijs, A new hybrid technology for planar fluxgate sensor fabrication, IEEE Trans. Magn. 35 (1999) 2111-2118.

[7] R. Boll, Soft Magnetic Materials, Vacuumschmelze, Hanau, 1993.

[8] Metglas ${ }^{\circledR}$ Product Literature, AlliedSignal, Morristown, NJ, USA, 1998.

[9] E. Belloy, M.A.M. Gijs, New etching-electroplating technology for quality factor enhancement of inductive devices,Proceeding no. 843, 8th European Conference on Power Electronics and Applications EPE'99, Lausanne, Switzerland, 1999.

\section{Biographies}

Eric Belloy was born in Geneva, Switzerland, in 1972. He received his diploma in Microengineering in 1997 from the Swiss Federal Institute of Technology of Lausanne (EPFL). His diploma work was the study, realisation, and characterisation of microtips on $\mathrm{Si}$ and glass substrates in use for the detection of the electrical activity of nerve cells. Since April 1997, he works as research assistant in the Institute of Microsystems at EPFL. He has first developed the technology process for a new magnetic sensor based on the Hall effect. Then, he has developed a new technology based on flex-foil patterning and assembling, in order to realise magnetic devices. His actual research area is on the dry-etching by micro-sandblasting.
Scott E. Gilbert received the $\mathrm{PhD}$ degree in physical chemistry from the University of California, Santa Barbara, in 1993. He then came immediately to the Swiss Federal Institute of Technology in Lausanne (EPFL) to postdoc at the Institute of Physical Chemistry for work on nanostructured liquid junction photovoltaic devices. Afterwards, he moved to the Department of Physics where his interests expanded to explore novel methods for ultra-thin metallic film and magnetic nanowire electrodeposition and electrical contacting methods. He is now with Prof. Gijs in the Institute of Microsystems as Project Leader for the development of now technologies for miniature inductive devices and electrochemical micromachining.

Olivier Dezuari was born in Lausanne, Switzerland, in 1972. He received the diploma in microengineering from the Swiss Federal Institute of Technology (EPFL), Lausanne, Switzerland, in 1997. From September 1997 to December 1998, he worked at the Institute of Microsystems at EPFL as Research Assistant, in the field of developing new technologies for the fabrication of inductive components, in particular flexible components.

Marco Sancho is a graduate student in Micro-engineering, who contributed to the present work during the course of a student research project.

Martin A.M. Gijs received his degree in Physics in 1981 from the Katholieke Universiteit Leuven, Belgium and his $\mathrm{PhD}$ degree in Physics at the same university in 1986. He joined the Philips Research Laboratories in Eindhoven, The Netherlands, in 1987. Subsequently, he has worked there on micro- and nano-fabrication processes of high critical temperature superconducting Josephson and tunnel junctions, the microfabrication of microstructures in magnetic multilayers showing the giant magnetoresistance effect, the design and realisation of miniaturised motors for hard disk applications, and the design and realisation of planar transformers for miniaturised power applications. He joined the Swiss Federal Institute of Technology Lausanne (Ecole Polytechnique Fédérale de Lausanne) in 1997 as a professor in the Institute of Microsystems of the Microengineering Department, where he is responsible for the Microsystems Technology group. His main interests are in developing technologies for novel inductive-type devices, new microfabrication technologies for microsystems fabrication in general and the development and use of microsystems technologies for biomedical applications in particular. 